\title{
Stationarity of the transition probabilities in the Markov chain formulation of owner payments on projects
}

\author{
Hanh $\operatorname{Tran}^{1} \quad$ David G. Carmichael ${ }^{2}$
}

(Received 9 January 2012; revised 11 April 2012)

\begin{abstract}
Markov chain theory has been used to model the likelihood of payment to contractors based on historical owner payment practices. An important assumption in this modelling of owner payment behaviour is that the transition probability matrices are stationary. We study this assumption: the null hypothesis postulated is that the transition matrices are stationary; the alternate hypothesis is that they are not. This article also explores the impact of this assumption on the performance of the model. The outcomes of the model, in the form of payment likelihood of an outstanding amount against its age, given by both stationary and non-stationary approaches are compared. Tests performed on two project data sets show that the null hypothesis is rejected at the $5 \%$ level of significance. However, the payment
\end{abstract}

http://journal austms.org.au/ojs/index.php/ANZIAMJ/article/view/5111 gives this article, (c) Austral. Mathematical Soc. 2012. Published April 28, 2012. ISSN 1446-8735. (Print two pages per sheet of paper.) Copies of this article must not be made otherwise available on the internet; instead link directly to this URL for this article. 
probabilities estimated using non-stationary transition matrices are shown to approach a steady state after a relatively short fluctuation. These steady state values of payment probabilities are almost identical to those estimated under a stationary assumption. Therefore, we recommend users of the model adopt the stationary transition matrix approach to avoid the extra mathematical complication caused by non-stationarity. This article reinforces the validity of the existing Markov chain formulation of owner payments and its assumption of stationarity. The analysis presented, although based on case study data, can be translated to any project, provided data in the right form are available.

\section{Contents}

1 Introduction

2 Background

2.1 Stationary testing of Markov chain transition matrices . . C72

2.2 Probability transition matrix in the Carmichael-Balatbat Markov chain formulation . . . . . . . . . . . . . .

3 Adaptation of the likelihood ratio test to the owner payment problem

3.1 Test procedure . . . . . . . . . . . . . . C75

3.2 Example: two lane, grade separated road . . . . . . . . C76

3.3 Example: steel fabrication . . . . . . . . . . . . . C78

4 Behaviour of non-stationary transition probability matrices 


\section{Introduction}

Markov chain theory is used in various applied fields and its modelling power has been demonstrated. Applications of the theory are found in engineering, chemistry, medicine, finance and other fields. In construction project management, Markov chain theory is used to model construction productivity [5] and owner payments [6]. The Markov chain formulation of owner payments [6] allows contractors to estimate the likelihood of late and incomplete payments in an uncertain payment environment. This formulation can be readily combined with the classification of typical owner payment behaviour $[23,24]$. The probability that an outstanding amount will be paid over time, or the probability that it may not be paid, feeds directly into the contractor's cash flow, cash planning, accounting and risk analysis practices.

The outcome of a Markov chain model depends on the probabilities within the transition matrix, and on whether the transition matrix is time independent (stationary) or time dependent (non-stationary). While a stationary assumption allows the straightforward calculation of the long-run equilibrium distribution of amounts in different states, changes in the transition probabilities over time may be a concern [11]. The assumption of stationarity in some established Markov chain models has been tested and rejected [8, 3, 25, e.g.].

In the Carmichael-Balatbat owner payment model [6], differences in claim sizes and the time at which claims are submitted are not taken into account, although it is suggested that they can be. Because claims and payments may occur over a relatively long period (maybe several years), they are subject to many external factors, such as project delays, the owner's financial status or administration procedures. The owner's payment of different claims, for example, between one submitted early in the project and one submitted near project completion, could be quite different in terms of timing and completeness. Hence, the transition probabilities near the project start may be different to those in the middle, or near the project end. Also, the owner's response to claims differing in size and type may also not be consistent 
(for example, a large claim may take a longer time to be assessed before being approved than a small claim). Consequently, the payment likelihood estimated using a stationary transition matrix may be different to that given by a non-stationary transition matrix.

This article examines the stationarity assumption in the Carmichael-Balatbat formulation, and the long run behaviour if non-stationary transition matrices are used. The article first summarises the established formulation of owner payment histories and available tests for stationarity. The theoretical likelihood ratio test is then specifically adapted to, and demonstrated on, actual owner payment data. Payment probabilities at a certain time following claim submission, obtained using a non-stationary approach, are then compared with those obtained using a stationary approach. The analysis is presented based on data from two projects, differing in size and type. Based on this comparison, conclusions and recommendations to the targeted users of the Carmichael-Balatbat formulation are given.

\section{Background}

\subsection{Stationary testing of Markov chain transition matrices}

Theoretical procedures for testing the stationarity of transition matrices are available. Of particular relevance to this article is the likelihood ratio test for stationarity [1]. In this test, the maximum likelihood estimates and their asymptotic distribution in a Markov chain of arbitrary order are obtained. There is also the $X^{2}$ test for homogeneity or fit [7, 16, e.g.], which is asymptotically equivalent to the likelihood ratio test [22]. Modifications of these two tests have been developed, for example, using Whittle's formula, $X^{2}$ distribution and maximum likelihood methods to estimate and test the parameters [4]; or using an extension of the Tsiasis model based on marginal 
distributions [20]. A method of stationarity testing by simple visual inspection of plots of transition probabilities over time was suggested by Kallberg and Saunders [14]. Barlett [2], Hoel [12] and Pardo [17], for example, developed mathematics of the estimation of non-stationary transition probabilities. Kiefer and Larson [15] and Weissbach, Tschiersch and Lawrenz [25] also discussed the calculation of non-stationarity transition probabilities.

Among the available test procedures, the likelihood ratio test appears to be used most commonly for verifying the stationarity assumption in applied Markov chain models $[21,13,3,25,26$, e.g.]. Test results vary from accepting to rejecting the hypothesis of stationarity, depending entirely on the data upon which the tests are performed. Some works accept the stationarity assumption [21, 13, e.g.]. Some works reject the stationarity assumption [3, $8,26,25$, e.g.]. The purpose of this article's analysis is similar to that of the above listed articles, but applied to the owner payment formulation [6].

\subsection{Probability transition matrix in the Carmichael-Balatbat Markov chain formulation}

The Carmichael-Balatbat Markov chain formulation of late and incomplete payments uses a summary of outstanding money against time after claim lodgement to calculate the transition probabilities between states. States are defined as the period of time by which payment is overdue. There are $\mathrm{n}$ transient states, $\mathrm{k}=0,1,2, \ldots, \mathrm{n}-1$, and two absorbing states, namely the 'To be resolved' and the 'Paid' states corresponding to $k=n, n^{\prime}$.

The transition probability, $p_{j k}$, is the probability associated with the transition of money from state $j$ to state $k$. Let $\alpha_{j k}$ be the amount in state $k$ that is transferred from state $j$ between periods $i$ and $i+1$ (this can be obtained directly from a summary of outstanding money against time after claim lodgement), then

$$
p_{j k}=\frac{\alpha_{j k}}{\sum_{k=0}^{n^{\prime}} \alpha_{j k}} .
$$


The $(n+2) \times(n+2)$ transition probability matrix $P$ contains the elements $p_{j k}$ where $j, k=0,1,2, \ldots, n, n^{\prime}[6]$.

Since movements only occur from a state to its immediate next state, or to state $n^{\prime}$, the matrix $P$ is only populated with elements $p_{j n^{\prime}}, p_{j, j+1}$ and $p_{n-1, n}$, $\mathfrak{j}=0,1,2, \ldots, \mathfrak{n}-1$. The matrix $P$ is arranged as

$$
\mathrm{P}=\left[\begin{array}{ll}
\mathrm{I} & \mathrm{O} \\
\mathrm{R} & \mathrm{Q}
\end{array}\right]
$$

where $\mathrm{I}$ is a $2 \times 2$ identity matrix, $\mathrm{O}$ is a $2 \times(n-1)$ zero matrix, $\mathrm{R}$ is an $(n-1) \times 2$ matrix and $Q$ is an $(n-1) \times(n-1)$ matrix. Matrices $I$ and $O$ always contain 1 and 0 elements, therefore, the following analysis only focuses on the entries of the matrices $\mathrm{R}$ and $\mathrm{Q}$.

The estimation of $\mathrm{P}$ in the above formulation is typically based on all project claims. That is, $\mathrm{P}$ is populated after payment of every claim has been made, or not made. An alternative to this is to estimate $\mathrm{P}$ at every claim entry in the claim list, based on the accumulating claims history. In this case, the entries of $\mathrm{P}$ at a time point $\tau$ reflect the ageing outstanding project money up to $\tau$. They also reflect the owner payment behaviour up to time point $\tau$. Another possible way to estimate the entries in the $\mathrm{P}$ matrix is by using the summary of all claims made within a certain project phase. Then, there is one matrix $P$ for each phase of the project and $\tau$ is taken to represent the phase number (for example, if the project has three phases then $\tau=1,2,3$ ). The last two methods of estimating the transition matrices are considered and tested in the next section. 


\section{Adaptation of the likelihood ratio test to the owner payment problem}

\subsection{Test procedure}

The likelihood ratio test, first given by Anderson and Goodman [1] and later demonstrated on real data [22], is adapted here to the owner payment case. Two actual project data sets demonstrate the test procedure.

The null hypothesis and the alternate hypothesis are:

$$
\begin{aligned}
& H_{0}: p_{j k}(\tau)=p_{j k} \quad(\tau=1,2, \ldots, s) ; \\
& H_{1}: p_{j k}(\tau) \text { is dependent on } \tau ;
\end{aligned}
$$

where $j, k$ are transient or absorbing states, $j, k=0,1,2, \ldots, n, n^{\prime}, \tau$ is a time point between project start and project end, and $s$ denotes project completion.

Under the alternate hypothesis $\mathrm{H}_{1}$, the transition probabilities at time point $\tau$, $p_{j k}(\tau)$, between periods $i$ and $i+1$, estimated at time point $\tau$, are

$$
p_{j k}(\tau)=\frac{\alpha_{j k}(\tau)}{\sum_{k=0}^{n^{\prime}} \alpha_{j k}(\tau)},
$$

where $\alpha_{j k}(\tau)$ is the amount in state $k$ that is transferred from state $j$ between periods $i$ and $i+1$, estimated at time point $\tau$.

The likelihood function maximised under $\mathrm{H}_{0}$ is

$$
L=\prod_{\tau}^{s} \prod_{j, k}^{n^{\prime}} p_{j k}^{\alpha_{j k}(\tau)} .
$$

The likelihood function maximised under $\mathrm{H}_{1}$ is

$$
L=\prod_{\tau}^{s} \prod_{j, k}^{n^{\prime}} p_{j k}(\tau)^{\alpha_{j k}(\tau)}
$$


Table 1: Payment profile -all claims; Example 3.2.

\begin{tabular}{|c|c|c|c|c|}
\hline $\begin{array}{l}\text { clair } \\
\text { amo } \\
(\$ \mathrm{~K})\end{array}$ & $\begin{array}{l}\text { utstanding } \\
1 \text { week }\end{array}$ & $\begin{array}{l}\text { Outstanding } \\
>2 \text { weeks } \\
(\$ \mathrm{~K})\end{array}$ & $\begin{array}{l}\text { Outstanding } \\
>3 \text { weeks } \\
(\$ \mathrm{~K})\end{array}$ & $\begin{array}{l}\text { Outstanding } \\
>4 \text { weeks } \\
(\$ \mathrm{~K})\end{array}$ \\
\hline & & $9,873.2$ & & $9,344.8$ \\
\hline
\end{tabular}

Thus the likelihood ratio, denoted as $\lambda$, is

$$
\lambda=\prod_{\tau}^{s} \prod_{j, k}^{n^{\prime}}\left(\frac{\hat{p}_{j k}}{\hat{p}_{j k}(\tau)}\right)^{\alpha_{j k}(\tau)} .
$$

The caret notation denotes a likelihood estimate. Anderson and Goodman [1] and Cramer [7] showed that $-2 \log (\lambda)$ is distributed as $X^{2}$ with $(s-1)(n+$ $2)(n+1)$ degrees of freedom when the null hypothesis is true (here $n+2$ is the total number of transition states). Since the distribution of $-2 \log (\lambda)$ is known, the probability of obtaining a value of $-2 \log (\lambda)$ or higher, if the hypothesis were true, can be determined. This conditional probability can be found using a table of the $X^{2}$ distribution under $(s-1)(n+2)(n+1)$ degrees of freedom.

\subsection{Example: two lane, grade separated road}

Project: construction of a $7 \mathrm{~km}$ two lane, grade separated road. A total of 41 progress claims were made over a total duration of 32 months. Under the original modelling using a stationary assumption [6], the transition probabilities are estimated based on a summary of total outstanding amounts for the entire project against weeks following claim submission. That is, the summary is made based on the full project data. Let $n=4$ and the time interval be a week. The payment profile summary is given in Table 1 . There are six states in total: states 0 to 3 corresponding to the amount owing beyond 0, 1, 2 and 3 weeks; state $n$ ' being the 'Paid' state; and state $n$ being the 'To be 
Table 2: Summary of outstanding claimed amounts in three project phases; Example 3.2.

\begin{tabular}{|c|c|c|c|c|c|}
\hline $\begin{array}{l}\text { Project } \\
\text { phase } \tau\end{array}$ & $\begin{array}{l}\text { Total } \\
\text { claimed } \\
\text { amount } \\
(\$ \mathrm{~K})\end{array}$ & $\begin{array}{l}\text { Outstanding } \\
>1 \text { week } \\
(\$ \mathrm{~K})\end{array}$ & $\begin{array}{l}\text { Outstanding } \\
>2 \text { weeks } \\
(\$ \mathrm{~K})\end{array}$ & $\begin{array}{l}\text { Outstanding } \\
>3 \text { weeks } \\
(\$ \mathrm{~K})\end{array}$ & $\begin{array}{l}\text { Outstanding } \\
>4 \text { weeks } \\
(\$ \mathrm{~K})\end{array}$ \\
\hline 1 & $12,089.9$ & $12,089.9$ & $12,089.9$ & $12,089.9$ & $7,416.0$ \\
\hline 2 & $40,110.5$ & $40,110.5$ & $40,110.5$ & $34,724.3$ & $5,186.2$ \\
\hline 3 & $62,363.4$ & $62,363.4$ & $57,672.8$ & $51,740.2$ & $6,742.6$ \\
\hline
\end{tabular}

resolved' state; $n=4$. Under the assumption of stationarity, the entries of the matrices $\mathrm{R}$ and $\mathrm{Q}$ were obtained directly from Table 1 and are

$$
\hat{\mathrm{R}}=\left\{\hat{\mathrm{r}}_{j k}\right\}=\left[\begin{array}{cc}
0 & 0 \\
0.041 & 0 \\
0.103 & 0 \\
0.804 & 0.196
\end{array}\right], \quad \hat{\mathrm{Q}}=\left\{\hat{\mathrm{q}}_{j k}\right\}=\left[\begin{array}{cccc}
0 & 1 & 0 & 0 \\
0 & 0 & 0.959 & 0 \\
0 & 0 & 0 & 0.897 \\
0 & 0 & 0 & 0
\end{array}\right] \text {. }
$$

Now allow the matrices $R$ and $Q$ to change with time throughout the project. For demonstration purposes, let the states be observed at three time points during the project: at the end of the first 11 months (phase 1), at the end of the next 10 months (phase 2) and at the end of the last 11 months (phase 3). That is, $\tau=1,2,3$. Table 2 summarises the outstanding amounts owing to the contractor in each of the phases. The payment profiles in Table 2 are summarised using claims made within a phase rather than all claims accumulated from the project beginning. The values of $\alpha_{j k}(\tau)$ for the three phases are derived directly from the payment data in Table 2. The transition probabilities between states from period $i$ to $i+1$, estimated at time point $\tau$, $p_{j k}(\tau)$, are calculated using Equation (3). The likelihood ratio $\lambda$ is calculated according to Equation (6),

$$
\lambda=\prod_{\tau}^{s} \prod_{j, k}^{n^{\prime}}\left(\frac{\hat{p}_{j k}}{\hat{p}_{j k}(\tau)}\right)^{\alpha_{j k}(\tau)}=\prod_{\tau}^{3} \prod_{j, k}^{n^{\prime}}\left(\frac{\hat{r}_{j k}}{\hat{r}_{j k}(\tau)} \cdot \frac{\hat{\mathrm{q}}_{j k}}{\hat{\mathrm{q}}_{j k}(\tau)}\right)^{\alpha_{j k}(\tau)} .
$$


Table 3: Summary of all claims; Example 3.3.

\begin{tabular}{|c|c|c|c|c|}
\hline $\begin{array}{l}\text { Total } \\
\text { claimed } \\
\text { amount }\end{array}$ & $\begin{array}{l}\text { Outstanding } \\
>2 \text { weeks } \\
(\$ \mathrm{~K})\end{array}$ & $\begin{array}{l}\text { Outstanding } \\
>4 \text { weeks } \\
(\$ \mathrm{~K})\end{array}$ & $\begin{array}{l}\text { Outstanding } \\
>6 \text { weeks } \\
(\$ \mathrm{~K})\end{array}$ & $\begin{array}{l}\text { Outstanding } \\
>8 \text { weeks } \\
(\$ \mathrm{~K})\end{array}$ \\
\hline $1,888.9$ & $1,888.9$ & 550.6 & 308.2 & 270.1 \\
\hline
\end{tabular}

Considering only nonzero elements, $\lambda=\left(1.595 \times 10^{-5}\right)^{10^{6}}$ and $-2 \log (\lambda)=$ $22.09 \times 10^{6}$. If the null hypothesis is true, the probability of observing a value of $-2 \log (\lambda)$ under $(s-1)(n+2)(n+1)$ degrees of freedom must be greater than 0.05 (for a one-sided test, $5 \%$ level of significance). Using a $X^{2}$ distribution table with 60 degrees of freedom, the probability of observing the above value of $-2 \log (\lambda)$ is far smaller than 0.05 . Thus there is strong evidence against the null hypothesis $\mathrm{H}_{0}$.

\subsection{Example: steel fabrication}

Project: steel fabrication. Twelve claims of total value of approximately $\$ 1.89 \mathrm{M}$ were submitted over a duration of 12 months. In this example an alternative way of establishing the $\mathrm{P}$ matrix over time, namely calculating $\mathrm{P}$ based on the to-date outstanding project money, is used. Let $n=4$ and the time interval be a fortnight. Then $P$ is a $6 \times 6$ matrix, $R$ is a $4 \times 2$ matrix and $\mathrm{Q}$ is a $4 \times 4$ matrix. Table 3 summarises the outstanding amounts against fortnights after claim lodgement, done for all claims combined. Now, for contrast, let the outstanding amounts be summarised each month during the 12 month project duration. At a certain month, the outstanding amounts are the actual to-date outstanding money, accumulated from the project start (Table 4). As anticipated, at $\tau=12$, the outstanding amounts are the same as those in Table 3, that is the $\mathrm{P}$ matrix with this approach eventually becomes the same as the $\mathrm{P}$ matrix obtained from Table 3. The likelihood ratio test procedure is repeated as above. The value of $\lambda$ is found to be 0.441 when 
Table 4: Summary by month of to-date outstanding money; Example 3.3.

\begin{tabular}{|c|c|c|c|c|c|}
\hline $\begin{array}{l}\text { At } \\
\text { month } \tau\end{array}$ & $\begin{array}{l}\text { Total } \\
\text { claimed } \\
\text { amount } \\
(\$ \mathrm{~K})\end{array}$ & $\begin{array}{l}\text { Outstanding } \\
>2 \text { weeks } \\
(\$ \mathrm{~K})\end{array}$ & $\begin{array}{l}\text { Outstanding } \\
>4 \text { weeks } \\
(\$ \mathrm{~K})\end{array}$ & $\begin{array}{l}\text { Outstanding } \\
>6 \text { weeks } \\
(\$ \mathrm{~K})\end{array}$ & $\begin{array}{l}\text { Outstanding } \\
>8 \text { weeks } \\
(\$ \mathrm{~K})\end{array}$ \\
\hline 1 & 89,531 & 89,531 & 89,531 & 89,531 & 89,531 \\
\hline 2 & 173,329 & 173,329 & 99,710 & 99,710 & 99,710 \\
\hline 3 & 421,008 & 198,096 & 124,477 & 124,477 & 124,477 \\
\hline 4 & 531,654 & 308,742 & 134,641 & 134,641 & 134,641 \\
\hline 5 & $1,082,009$ & 344,784 & 170,683 & 170,683 & 170,683 \\
\hline 6 & $1,358,504$ & 407,462 & 233,361 & 233,361 & 233,361 \\
\hline 7 & $1,657,696$ & 440,712 & 266,611 & 266,611 & 266,611 \\
\hline 8 & $1,709,917$ & 492,933 & 267,075 & 267,075 & 267,075 \\
\hline 9 & $1,801,566$ & 492,933 & 267,075 & 267,075 & 267,075 \\
\hline 10 & $1,818,082$ & 509,449 & 267,075 & 267,075 & 267,075 \\
\hline 11 & $1,847,810$ & 509,449 & 267,075 & 267,075 & 267,075 \\
\hline 12 & $1,888,942$ & 550,581 & 308,207 & 270,101 & 270,101 \\
\hline
\end{tabular}

the amounts are in thousands of dollars. Therefore $-2 \log (\lambda)=1636.1$. The probability of observing a value of 1636.1 under $6 \times 5 \times 11=330$ degrees of freedom is smaller than 0.05 , and therefore there is strong evidence against the null hypothesis. The test result stays the same when the amounts are in dollars or million of dollars.

The test results in the two examples show that the assumption of stationarity is not valid for the owner payment problem. The following section looks at the behaviour of a non-stationary $\mathrm{P}$ matrix throughout the project and the difference in the prediction accuracy between assuming stationarity and non-stationarity. 


\section{Behaviour of non-stationary transition probability matrices}

The change in the calculated payment likelihood due to changes in the entries of the (non-stationary) transition probability matrix along the project is of interest. This section examines the time dependent payment likelihood obtained by allowing $P(\tau)$ to change after every claim entry. The steady state of $P(\tau)$ and its rate of approaching this steady state are ascertained.

Let $\mathrm{P}(\tau)$ be calculated from the summaries of the accumulating outstanding project money against the age of claims made up to time point $\tau$. The newly calculated transition probabilities are then substituted for the constant probabilities in the Carmichael-Balatbat model. The evolving probabilities of being in state $k, k=n, n^{\prime}, 0,1,2, \ldots, n-1$, denoted by vector $\pi$, are used as an indicator of the time dependent behaviour of $\mathrm{P}(\tau)$. Data from the two Examples 3.2 and 3.3 are used to demonstrate the results.

Compared to the stationary case, modelling with non-stationary transition probabilities is far more complicated while the generalisation power is less. Every entry of $\mathbf{P}$, and hence every model output is a function of time [9], and largely depends on the specific data being used. The changing entries in $\mathrm{P}(\tau)$ can also be difficult to establish. Existing literature focuses on the estimation of the non-stationary transition probabilities (especially when they are not observable), their convergence (if any) and the rate of convergence [10, 19, 9, e.g.]. In the owner payment case, the non-stationary transition matrices are observable. Still, there is no general solution to the equation $\pi=\pi \mathrm{P}$, and therefore the analysis by Carmichael and Balatbat [6] cannot be generalised to all claims submitted at any time point along the project. It is only true for one particular claim submitted at the time point (or phase) $\tau$ at which the transition matrix $P(\tau)$ is estimated and used in the calculations. The analysis does not need to be redone; however, its generalisation power is lost. The initial state probability vector for a newly submitted claim is $\boldsymbol{\pi}=$ 
$[0,0,1,0, \ldots, 0]$; that is, there is probability 1 of being in the transient state 0 . The first entry of the vector $\boldsymbol{\pi}$, which corresponds to the probability of being in state $\mathrm{n}^{\prime}$, is the probability that the amount is paid. Under the assumption of stationarity, after one time period, the probabilities of that amount being in states $\mathrm{k}$ are $\pi \mathrm{P}$, after two time periods $\pi \mathrm{P}^{2}$, after three time periods $\pi \mathrm{P}^{3}$, and so on. Under the assumption of non-stationarity, if a claim is submitted at time point $\tau$ and $P(\tau)$ is the transition probability matrix estimated at that time, then after one time period $\pi$ changes to $\pi \mathrm{P}(\tau)$, after two time periods $\pi$ changes to $\pi \mathrm{P}(\tau) \mathrm{P}(\tau+1)$, after three time periods $\pi$ changes to $\pi \mathrm{P}(\tau) \mathrm{P}(\tau+1) \mathrm{P}(\tau+2)$, and so on. In general, the vector $\pi$ satisfies [19]

$$
\boldsymbol{\pi}_{\mathrm{T}}=\boldsymbol{\pi}_{\tau} \prod_{\mathrm{r}=\tau}^{\mathrm{T}-1} \mathrm{P}(\mathrm{r}), \quad \mathrm{T}=\tau+1, \tau+2, \ldots
$$

Consider Example 3.3. Under a stationary assumption, the probabilities of a claim being paid in the first, second, third and fourth time periods (fortnights) after claim lodgement are 0, 0.709, 0.837 and 0.857, respectively. Under a non-stationary assumption, if the claim is made in the first month of the project then the probability of it being paid after one to four time periods evolves as 0, 0, 0.372 and 0.372 , which is far different from those obtained in the stationary scenario. However looking further ahead in time, if the claim is made in the ninth month (a time closer to project completion) then the probabilities of it being paid in the first, second, third and fourth fortnights are 0, 0.720, 0.853 and 0.871, which are almost the same as those calculated in the stationary case. That is, towards the end of the project, when the non-stationary matrix $\mathrm{P}(\tau)$ approaches the stationary matrix $\mathrm{P}$, the entries of vector $\pi$ also approach those of the stationary case.

In order to identify the point at which the entries of $\pi$ given by $\mathrm{P}(\tau)$ are close enough to those given by $P$, the entries of $\boldsymbol{\pi}$ are plotted for the same unit claim made at different times $\tau$ along the project timeline. The entries of $\boldsymbol{\pi}$ for the non-stationary case are calculated at every time point $\tau=1,2, \ldots, 9$ using $\mathrm{P}(\tau)$. For demonstration purposes, the probability that an amount being 


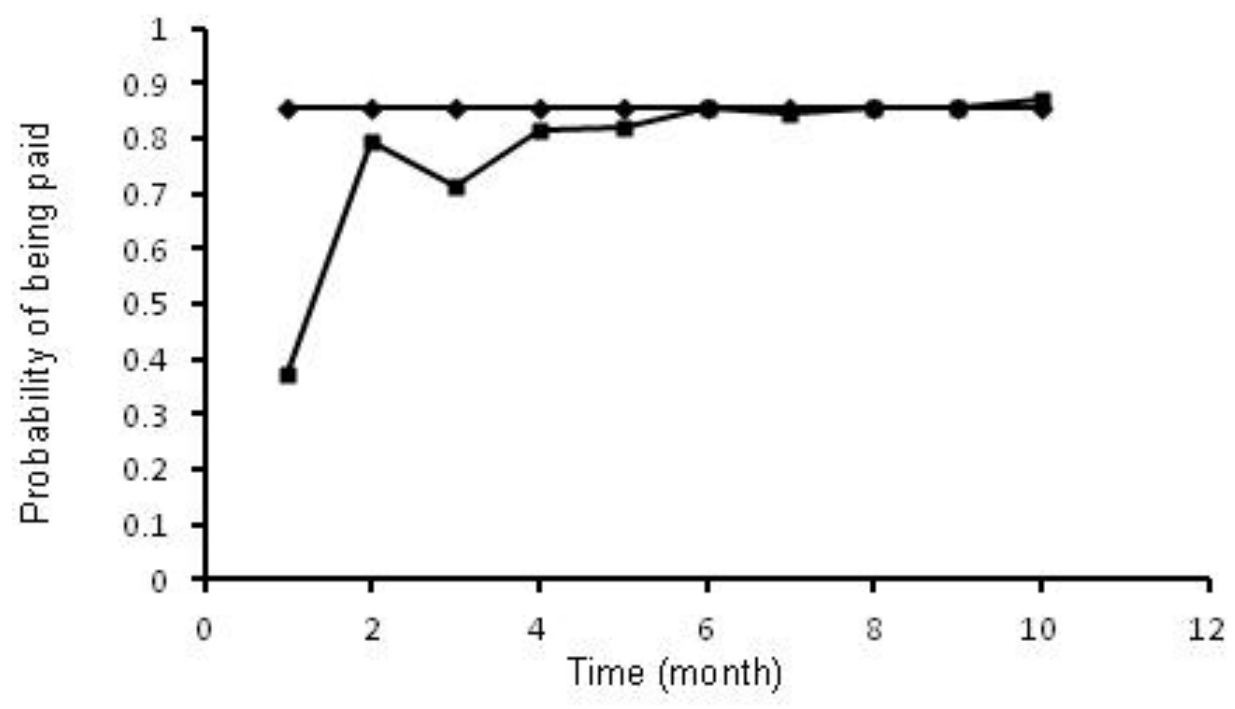

$\rightarrow$ Stationary $\rightarrow-$ Non-stationary

Figure 1: Probability of being paid at four fortnights following claim lodgement. Stationary and non-stationary approaches. Example 3.3.

paid four fortnights after its submission, using the two approaches, is shown in Figure 1. The horizontal axis shows the month $(\tau)$ in which the claim is submitted, different months result in different values of $\pi \mathrm{P}(\tau) \mathrm{P}(\tau+1) \mathrm{P}(\tau+2)$.

Of 12 claims in total, Figure 1 shows that the probability of a claim made in the first, second, third or fourth month being paid after four fortnights estimated using the two approaches is quite different. However, from month five onwards, the estimation results using non-stationary and stationary approaches are almost identical. It only takes a few time periods before the results obtained based on a stationary assumption become as accurate as those obtained by a non-stationary approach.

Consider Example 3.2. Because the first seven claims were not paid, the first entry of $\pi$ remains at zero if a new claim occurs in the first seven 


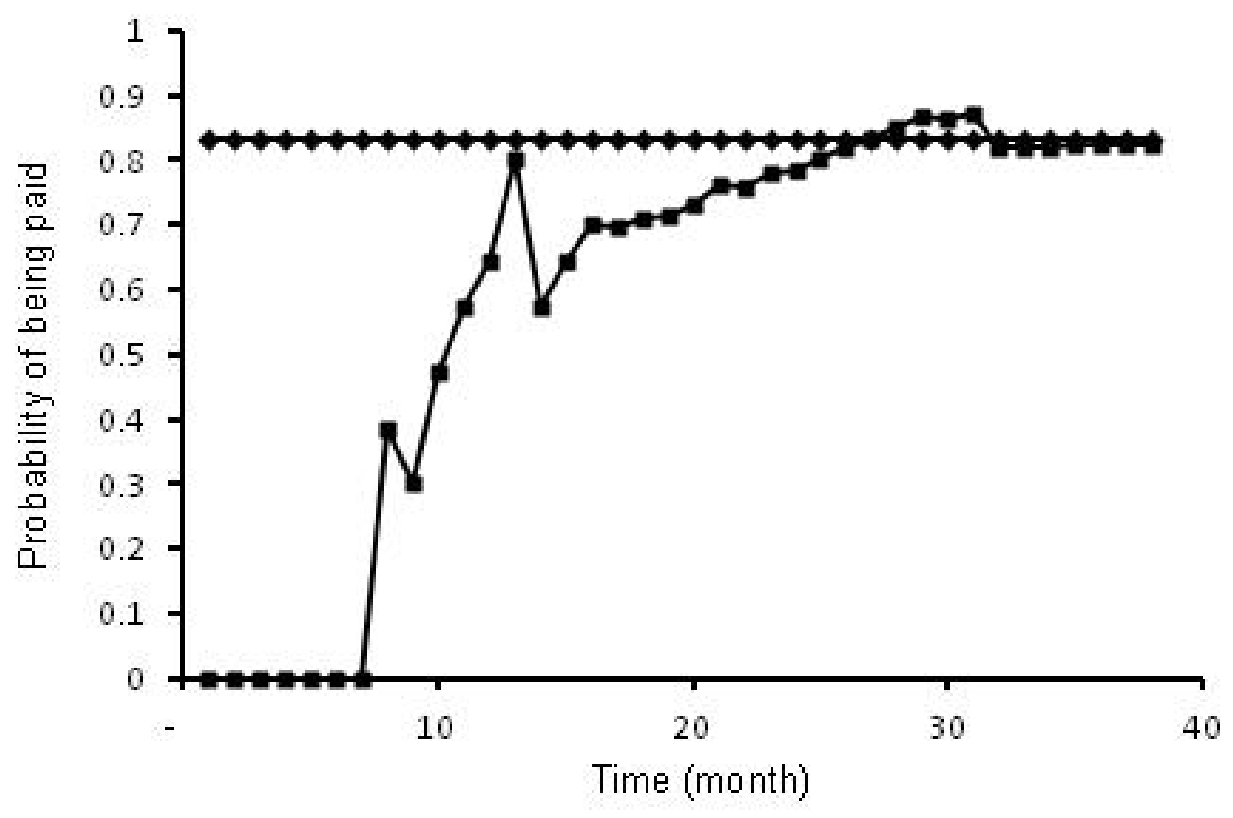

$\rightarrow$ Stationary $\rightarrow$ Non-stationary

Figure 2: Probability of being paid at four weeks following claim lodgement. Stationary and non-stationary approaches. Example 3.2.

months. If a new claim is made in months eight, nine or ten, then the difference in the evolving first entry of $\boldsymbol{\pi}$ between the non-stationary and stationary approaches is very large. From month 17 onwards, the values of $\pi \mathrm{P}^{3}$ and $\pi \mathrm{P}(\tau) \mathrm{P}(\tau+1) \mathrm{P}(\tau+2)$ become almost the same. The first entries of $\boldsymbol{\pi}$ under stationary and non-stationary assumptions are shown in Figure 2.

Large claims and the time at which they are made can strongly influence the values in $\mathrm{P}(\tau)$. Large-valued claims dominate $\mathrm{P}(\tau)$ over small claims; for example, if a large claim was submitted but was not paid (within the defined time allowance) then the value of $\mathrm{P}(\tau)$ before and after this claim entry may be quite different. In Example 3.3, $\mathrm{P}(\tau)$ reaches its steady state after four 
claims (of 12 in total) partly because the large claims (in a relative sense) were mostly submitted at the beginning. In Example 3.2, the larger claims are submitted in the second half of the project, leading to a longer fluctuation period ( 16 out of 41 claims) before $P(\tau)$ reaches its steady state.

In both case examples, it only takes less than half of the claim list for the nonstationary transition matrix to reach a steady state position where the entries in $\mathrm{P}(\tau)$ become stable and very close to those estimated under a stationary assumption. That is, the long-run steady state transition probabilities are reached relatively quickly. On this basis, it is considered that the extra time and complication in computing a non-stationary $\mathrm{P}(\tau)$ is not worthwhile.

\section{Conclusion}

The article gives a validation, for application purposes, of the assumption of stationarity in the Markov formulation of owner payments. Likelihood ratio tests were performed to test the hypothesis that $\mathrm{P}$ is constant over time against the alternate hypothesis that $P$ is time dependent. Two case studies were used for testing. The tests found that the null hypothesis is rejected at the 5\% level of significance in both cases, implying that the assumption of stationarity does not hold for the owner payments formulation for these two data sets.

Despite the rejection of the stationarity hypothesis, further comparison between the model outputs given by stationary and non-stationary transition matrices showed that the stationary approach still yields acceptable accuracy for a contractor's estimation purposes. Non-stationary transition matrices were calculated at every claim entry in a project's claim list. The time dependent behaviour of $P(\tau)$ was then illustrated through the evolution of the probability of a claim being paid in the subsequent time periods after claim submission (vector $\pi$ ). It was shown that $P(\tau)$ reaches a steady state after approximately one third of the claim list and the steady state values 
are within the $\pm 10 \%$ range of those given by using a stationary $P$ matrix. This implies similar outputs are produced by stationary and non-stationary transition matrices in the later two thirds of the projects.

When doing the analysis using the non-stationary transition matrix approach, a contractor could expect fluctuation in the entries of the $\mathrm{P}(\tau)$ matrix before a steady state is reached. In terms of payment likelihood, it is anticipated that the vector $\boldsymbol{\pi}$ for claims in the early part of a project using a stationary $P$ matrix will differ from those estimated using a non-stationary $P(\tau)$. In many cases, claims made earlier in a project are lower than those made later, and the discrepancy in results, between stationary and non-stationary assumptions, in the early part of a project will be small. For the later two thirds of a project, there is almost no difference between payment likelihood estimated using stationary or non-stationary assumptions.

These conclusions were derived based on two case study projects of different types and sizes. However, the presented test procedure and analysis could be extended to other project data sets. Further research could examine the time dependent behaviour of the transition matrices by expressing the transition probabilities as functions of time. Accordingly, the Carmichael-Balatbat formulation could be modified to incorporate time as a variable.

For application purposes, we recommend a contractor assume that the P matrix is constant over the entire project when estimating payment probabilities. The entries of $\mathrm{P}$ should be calculated using a payment profile, which is a summary of total outstanding project money against time passing since claim lodgement. The summary can be done for all claims, or for all claims of one type (for example, progress claims, variation claims or extension of time claims). This approach is straightforward and practical, yet of sufficient accuracy. 


\section{References}

[1] T. W. Anderson and L. A. Goodman, Statistical Inference about Markov Chains. Annals of Mathematical Statistics, Vol. 28, No. 1, pp. 89-110, 1957. doi:10.1214/aoms/1177707039 C72, C75, C76

[2] M. S. Barlett, The frequency goodness of fit test for probability chains. Proceedings of the Cambridge Philosophical Society, Vol. 47, No. 1, pp. 86-95, 1951. doi:10.1017/S0305004100026402 C73

[3] L. Betancourt, Using Markov chains to estimate losses from a porforlio of mortgages. Review of Quantitative Finance and Accounting, Vol. 12, No. 3, pp. 303-317, 1999. doi:10.1023/A:1008331016892 C71, C73

[4] P. Billingsley, Statistical methods in Markov chains. Annals of Mathematical Statistics, Vol. 32, No. 1, pp. 12-40, 1961. doi:10.1214/aoms/1177705136 C72

[5] D. G. Carmichael, Engineering Queues in Construction and Mining. Ellis Horwood Ltd. (John Wiley and Sons Ltd.), Chichester, 1987. C71

[6] D. G. Carmichael and M. C. A. Balatbat, A contractor's analysis of the likelihood of payment of claims. Journal of Financial Management of Property and Construction, Vol. 15, No. 2, pp. 102-117, 2010. doi:10.1108/13664381011063412 C71, C73, C74, C76, C80

[7] H. Cramer, Mathematical methods of statistics. Princeton University Press, Princeton, New Jersey, 1946 C72, C76

[8] B. D. Fielitz, On the stationarity of transition probability matrices of common stocks. Journal of Financial and Quantitative Analysis, Vol. 10, No. 2, pp. 327-339, 1975. doi:10.2307/2979039 C71, C73

[9] F. Hahary, N. Lipstein and G. P. H. Styan, A matrix approach to nonstationary chains. Operations Research, Vol. 18, No. 6, pp. 1168-1181, 1970. doi:10.1287/opre.18.6.1168 C80 
[10] M. C. Hallberg, Projecting the size distribution of agricultural firms. An application of a Markov process with non-stationary transition probabilities. American Journal of Agricultural Economics, Vol. 51, No. 2, pp. 289-302, 1969. doi:10.2307/1237580 C80

[11] M. Hierro and A. Maza, Non-stationary transition matrices: An overlooked issue in intra-distribution dynamics. Economics Letters, Vol. 103, No. 2, pp. 107-109, 2009. doi:10.1016/j.econlet.2009.02.005 C71

[12] P. G. Hoel, A test for Markoff chains. Biometrika, Vol. 413, No. 3/4, pp. 430-433, 1954. http://www.jstor.org/stable/2332723 C73

[13] S. Jain, Markov chain model and its application. Computers and Biomedical Research, Vol. 19, No. 4, pp. 374-378, 1986. doi:10.1016/0010-4809(86)90049-2 C73

[14] J. G. Kallberg and A. Saunders, Markov chain approaches to the analysis of payment behaviour of retail credit customers. Financial Management, Vol. 12, No. 2, pp. 5-14, 1983.

http://www.jstor.org/stable/3665204 C73

[15] N. M. Kiefer and C. E. Larson, Testing statistical hypotheses. John Wiley and Sons, New York, 1959. C73

[16] E. L. Lehmann, Testing statistical hypotheses. John Wiley and Sons Inc., New York, 1959. C72

[17] M. C. Pardo, Testing stationary distributions of Markov chains based on Rao's divergence. Applied Mathematics Letters, Vol. 12, No. 1, pp. 31-36, 1999. doi:10.1016/S0893-9659(98)00122-0 C73

[18] E. Parzen, Stochastic Processes.Holden-Day Inc., San Francisco, 1962

[19] M. S. Salkin, R. E. Just and O. A. Cleveland, Estimation of nonstationary transition probabilities for agricultural firm size 
projection. The Annals of Regional Science, Vol. 10, No. 1, pp. 71-82, 1976. doi:10.1007/BF01291236 C80, C81

[20] M. Z. Sirdari, M. A. Islam and N. Awang, A stationary test on Markov chain models based on marginal distribution. Proceedings of the 6th IMT-GT Conference on Mathematics, Statistics and its Applications, Kuala Lumper, Malaysia, 2010.

http://research . utar . edu . my/CMS/ICMSA2010/ICMSA2010_ Proceedings/files/statistics/ST-Zang.pdf C73

[21] G. P. H. Styan and H. Smith, Markov chains applied to marketing. Journal of Marketing Research, Vol. 1, No. 1, pp. 50-55, 1964. http://www. jstor.org/stable/3150320 C73

[22] M. Tainiter, Esimation, hypothesis testing and parameter correlation for Markov chains. Transaction on Reliability, Vol. R-12, No. 4, pp. 26-36, 1963. doi:10.1109/TR.1963.5218225 C72, C75

[23] H. Tran, D. G. Carmichael and M. C. A. Balatbat, Tree form classification of owner payment behaviour. Proceedings of the 4 th International Conference on Construction Engineering and Project Management, Sydney, Australia, 2011. C71

[24] H. Tran and D. G. Carmichael, A contractor's classification of owner payment practices. Engineering, Construction and Architectural Management, accepted for publication, 2012. C71

[25] R. Weissbach, P. Tschiersch and C. Lawrenz, Testing time-homogeneity of rating transtions after origination of debt. Empirical Economics, Vol. 36, No. 3, pp. 575-596, 2009. C71, C73

[26] R. Weissbach and R. Walter, A likelihood ratio test for stationarity of rating transitions. Journal of Econometrics, Vol. 155, No. 2, pp. 188-194, 2010. doi:10.1016/j.jeconom.2009.10.016 C73 


\section{Author addresses}

1. Hanh Tran, School of Civil and Environmental Engineering, The University of New South Wales, Australia. mailto:hanh.tran@student.unsw. edu.au

2. David G. Carmichael, School of Civil and Environmental Engineering, The University of New South Wales, Australia. mailto:d.carmichael@unsw.edu.au 